\title{
Ab Initio Study of Acceptor-Donor Complexes in Silicon and Germanium
}

\author{
A. Settels, T. Korhonen,* N. Papanikolaou, R. Zeller, and P. H. Dederichs \\ Institut für Festkörperforschung, Forschungszentrum Jülich, D-52425 Jülich, Germany
}

(Received 30 June 1999)

\begin{abstract}
The electronic and geometrical structures, in particular the electric field gradients (EFGs), of $[\mathrm{Cd} D]^{-}$ $(D=\mathrm{P}, \mathrm{As}, \mathrm{Sb})$ acceptor-donor pairs in $\mathrm{Si}$ and $\mathrm{Ge}$ are studied using the full potential Korringa-KohnRostoker Green's function method. In addition, also neutral complexes $\left([\mathrm{Cd} D]^{0}\right)$ and trimers $\left(\left[\mathrm{Cd} D_{2}\right]^{0}\right)$ in $\mathrm{Si}$ are investigated. The EFG depends very sensitively on the large lattice relaxations induced by the defects and can be understood by a simple hybridization model. Our calculations are in good agreement with experimental results and provide a consistent picture of acceptor-donor complexes in Si and $\mathrm{Ge}$.
\end{abstract}

PACS numbers: 71.55.Cn, 61.72.Tt, 76.60.-k

The donor-acceptor pairs in silicon and germanium have attracted intensive research interest for a long time [15]. If impurities with opposite charges are either intentionally or unintentionally introduced in semiconductors, the Coulomb attraction leads to the formation of donoracceptor complexes which can cause serious technological problems such as an uncontrolled annihilation or creation of charge carriers. To study the behavior of these defects, mostly nuclear techniques such as perturbed $\gamma \gamma$ angular correlation (PAC) experiments were successfully applied to obtain microscopic information on an atomic scale. A very popular and well-known probe atom is the radioactive ${ }^{111} \mathrm{In} /{ }^{111} \mathrm{Cd}$ nucleus. In these experiments initially a dimer complex is formed consisting of the In acceptor and a donor atom such as $\mathrm{P}, \mathrm{As}$, or $\mathrm{Sb}$, so that neutral $[\operatorname{In} D]^{0}$ $(D=\mathrm{P}, \mathrm{As}, \mathrm{Sb})$ pairs are produced. Since such neutral pairs have no states in the gap and thus are not magnetically or electrically active, very little information can be obtained with other experimental methods. After the decay ${ }^{111} \mathrm{In} \rightarrow{ }^{111} \mathrm{Cd}$, the PAC measurement takes place at the daughter complex $\mathrm{Cd} D$. The fact that the electric field gradient is independent from the $\operatorname{In} D$ mother complex has been proven by PAC experiments starting from the ${ }^{111 m} \mathrm{Cd}$ nucleus as mother probe atom [3]. Because of the pair formation the cubic surrounding of the probe atom ${ }^{111} \mathrm{Cd}$ is disturbed, leading to an electric field gradient (EFG) at the $\mathrm{Cd}$ site which can be determined in particular for nonmagnetic systems. This "fingerprint" of the defect system gives some important information about the defect structure. For instance, the main axis of the EFG is found to point along the [111] direction $(\eta=0)$, which strongly suggests a nearest neighbor (nn) pair configuration. However, for a fundamental understanding of the complexes electronic structure calculations are needed [3], which can be performed with good precision by density-functional methods [6,7]. The use of pseudopotential methods, which usually give excellent results in semiconductor physics, is not suitable in this case since the EFG is determined by the $p$ charge density in the inner core region [6], so that up to now no theoretical treatment of these fundamental acceptor-donor complexes in $\mathrm{Si}$ and Ge exists.
In this paper we calculate the electronic and geometrical structures and the EFGs of singly negatively charged defect pairs $[\mathrm{Cd} D]^{-}(D=\mathrm{P}, \mathrm{As}, \mathrm{Sb})$ in $\mathrm{Si}$ and Ge. Furthermore, we show that also neutral $[\mathrm{Cd} D]^{0}$ pairs exist which is in agreement with experiments [5]. We also investigate trimer complexes $\left[\mathrm{Cd} D_{2}\right]^{0}$ (with $D=\mathrm{P}$, As). Our calculations provide a consistent picture of the physical properties of acceptor-donor complexes in $\mathrm{Si}$ and Ge.

The theoretical approach is based on the densityfunctional theory in the local density approximation (LDA) parametrized by Vosko et al. [8]. The calculations are done with the multiple scattering Korringa-KohnRostoker (KKR) Green's function method [9-11]. In this method the perturbed region around the defect complexes is exactly embedded in the unperturbed crystal by using Green's function technique. In this all-electron method, the fundamental density-functional quantity, the electron density, is calculated by a contour integral in the complex energy plane. We have divided the energy contour into two parts covering the energy region of the valence bands and the gap states separately, so that we can arbitrarily specify the occupancy of the gap states.

In our calculations we use the LDA lattice constants (for $\mathrm{Si}, 5.40 \AA$ and, for $\mathrm{Ge}, 5.57 \AA$ ) as calculated in [12] and describe the diamond lattice structure using four basis positions along the [111] direction, two for the $\mathrm{Si}$ or Ge atoms and two empty sites. These empty sites facilitate the $l$ convergence in our full-potential KKR calculations [12], where no atomic sphere approximations for the potential, for the charge, nor for the WignerSeitz cell are used. For the substitutional nn pairs along the [111] direction a cluster of 76 perturbed potentials (38 atoms) with $C_{3 v}$ symmetry was embedded in the unperturbed ideal host crystal, whereas the substitutional $\mathrm{Cd} D_{2}$ complexes were calculated along the [110] "zigzag" line within a cluster of 84 perturbed potentials (40 atoms) exploiting the $C_{2 v}$ symmetry.

We determine the equilibrium positions of the atoms by the condition of vanishing forces, which was tested to be equivalent to the condition of minimal total energy. The forces are calculated from the charge density using the 
so-called "ionic" Hellmann-Feynman theorem [13]. Since the positions in the perturbed crystal differ from the ones in the ideal host the corresponding host Green's function and the potentials have to be transformed to the new shifted positions, as described in Ref. [13]. We truncate spherical harmonics expansions of the Green's function and wave functions with a cutoff $l_{\max }=4$, which leads to a potential and charge density cutoff $2 l_{\max }$. In deviation from [13] we replace the cellular division of space as determined by the host lattice by a Voronoi construction, such that the shifted atoms are located at the center of the new cells. For large atoms this significantly improves the $l$ convergence [14].

$[C d D]^{-}$complexes in $\mathrm{Si}$ and Ge.-The calculated EFGs for the negatively charged $[\mathrm{Cd} D]^{-}$complexes in $\mathrm{Si}$ and $\mathrm{Ge}$ and for the neutral $[\mathrm{Cd} D]^{0}$ pairs in $\mathrm{Si}$ are displayed in Table I for the ideal and the relaxed configurations in comparison with experimental results. The nuclear coupling constant $\nu$ at the $\mathrm{Cd}$ site is calculated from the largest component of the electric field gradient tensor $V_{z z}$ via $\nu=\frac{e Q}{h} V_{z z}$, where the nuclear quadrupole moment of the ${ }^{111} \mathrm{Cd}$ probe atom is assumed to be $Q=0.83 \mathrm{~b}$. The assumed $C_{3 v}$ symmetry yields a vanishing asymmetry parameter, $\eta$, which is in agreement with experiments. The calculated lattice relaxations for the $[\mathrm{Cd} D]^{-}(D=\mathrm{P}, \mathrm{As}, \mathrm{Sb})$ pairs in silicon and their nearest Si-neighbors are shown in Fig. 1. The EFG depends very sensitively on the relaxations of the atoms. Without lattice relaxations, the calculation of EFGs is meaningless leading even to a wrong trend. For the relaxed positions, the agreement between theory and experiment is quite good for both $\mathrm{Si}$ and $\mathrm{Ge}$. As it can be seen from Fig. 1, the lattice distortions - especially at the $\mathrm{Cd}$ sitestrongly increase with increasing donor size. Compared to that, the relaxations for the same defect pairs in germanium are somewhat smaller, but the general trend (increasing relaxations with increasing donor size) remains. For instance, for $[\mathrm{CdP}]^{-}$in $\mathrm{Ge}$ the $\mathrm{Cd}$ atom relaxes $0.8 \%$

TABLE I. The quadrupole coupling constant at the $\mathrm{Cd}$ site, $\nu=e Q_{\mathrm{Cd}} V_{z z} / h$, calculated using a nuclear quadrupole moment $Q_{\mathrm{Cd}}=0.83 \mathrm{~b}$ and the largest component, $V_{z z}$, of the EFG tensor. The sign is that of $V_{z z}$.

\begin{tabular}{lccc}
\hline \hline & $\mathrm{EFG}_{\text {ideal }}$ & $\mathrm{EFG}_{\text {relaxed }}$ & $\mathrm{EFG}_{\text {exp }}[1-5]$ \\
\hline & \multicolumn{3}{c}{ Si host } \\
{$[\mathrm{CdP}]^{-}$} & -189 & -191 & \pm 179 \\
{$[\mathrm{CdAs}]^{-}$} & -174 & -249 & \pm 229 \\
{$[\mathrm{CdSb}]^{-}$} & -85 & -294 & \pm 271 \\
{$[\mathrm{CdP}]^{0}$} & -79 & -56 & \pm 36 \\
{$[\mathrm{CdAs}]^{0}$} & -73 & -89 & \pm 83 \\
{$[\mathrm{CdSb}]^{0}$} & +6 & -135 & \pm 129 \\
& & $\mathrm{Ge}$ host & \\
{$[\mathrm{CdP}]^{-}$} & -135 & -124 & \pm 86 \\
{$[\mathrm{CdAs}]^{-}$} & -118 & -182 & \pm 148 \\
{$[\mathrm{CdSb}]^{-}$} & +2 & -235 & \\
\hline \hline
\end{tabular}

of the nn distance inward and the $\mathrm{P}$ atom only $2.9 \%$ of the $\mathrm{nn}$ distance outward. By exchanging $\mathrm{P}$ with As the outward movement of the $\mathrm{Cd}$ atom increases to $2.4 \%$, while the As atom shifts outward by $2.4 \%$. In the case of the $[\mathrm{CdSb}]^{-}$pair the $\mathrm{Cd}$ atom relaxes by $11.5 \%$ outwards and the $\mathrm{Sb}$ atom by $1.7 \%$ inwards, respectively. Consequently for the $\mathrm{CdSb}$ pair one expects again the biggest change in the EFG compared to the ideal configuration as can be seen from Table I.

Since we reproduced the experimental results and gave a description of the geometrical arrangements, our goal is now to attain more insight into the origin of the EFG by a detailed analysis of the electronic structure. The main contribution to the EFG is given by the quadrupole component of the charge density, which is mainly determined by the $p-p$ anisotropy contribution of the Green's function [6]. Because of the [111] symmetry axis along the defect pair we choose this direction to be the $z$ axis of our coordinate system. Then the EFG along the [111] direction is proportional to the anisotropy of the $p$ charge of the Cd atom: $V_{z z} \propto \int^{E_{F}}\left\{\rho_{p z}(E)-\right.$ $\left.\frac{1}{2}\left[\rho_{p x}(E)+\rho_{p y}(E)\right]\right\} d E[6]$. The projections of the local $p$ density of states along the [111] direction, $\rho_{p z}(E)$, and perpendicular to [111], $\rho_{p x}(E)=\rho_{p y}(E)$, are shown in Fig. 2. The $p_{z}$ orbital belongs to the fully symmetrical $A_{1}$ subspace of the $C_{3 v}$ symmetry group, while the $p_{x}$ and $p_{y}$ orbitals belong to the $E$ subspace. If the charge density around the $\mathrm{Cd}$ site is symmetric, as is the case for an isolated substitutional $\mathrm{Cd}$ atom, the $p$ charge density is isotropic $\left(\rho_{p x}=\rho_{p y}=\rho_{p z}\right)$ leading to a vanishing EFG $V_{z z}$. The origin of the EFG can now be understood

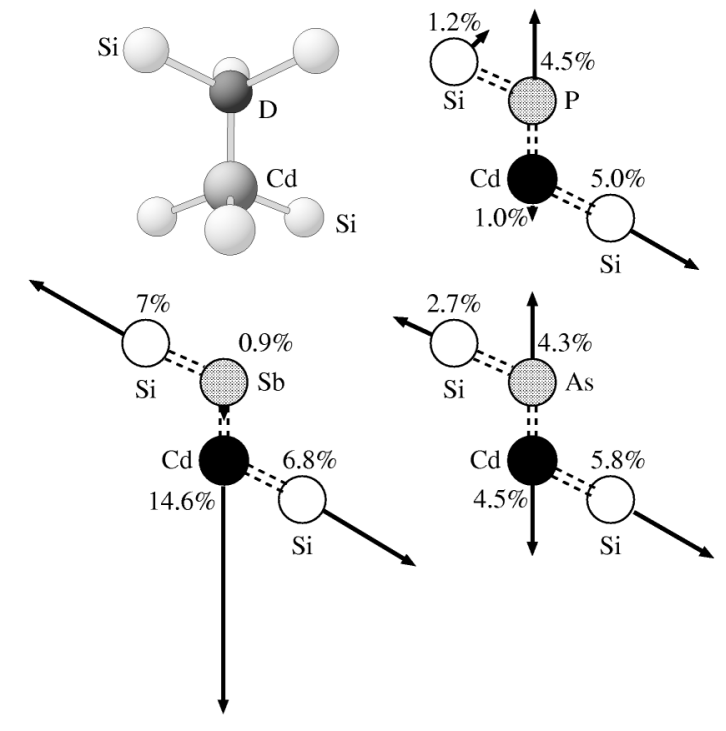

FIG. 1. Lattice relaxations for the defect atoms and the nn atoms of $[\mathrm{Cd} D]^{-}(D=\mathrm{P}, \mathrm{As}, \mathrm{Sb})$ defect pairs in Si. Shown is the three-dimensional arrangement of the $\operatorname{Cd} D$ complex (top, left hand) and the (110) planes containing the $\operatorname{Cd} D$ pairs and the two representative Si neighbors. Relaxations are given in percentage of the nn distance. 


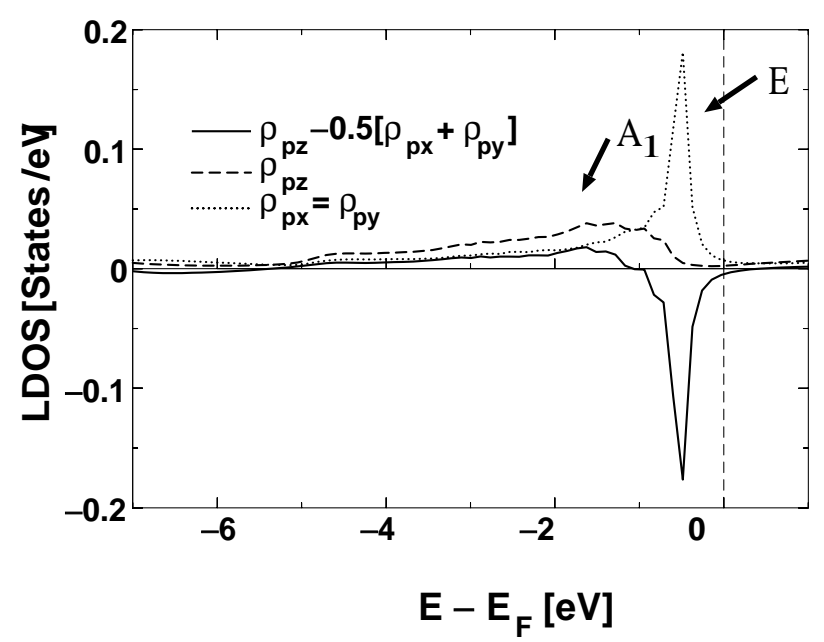

FIG. 2. The $p$-orbital local density of states (LDOS) of the $\mathrm{CdAs}$ pair in $\mathrm{Si}$ at the $\mathrm{Cd}$ site. The solid line represents the anisotropy of the local $p$ density of states, $\rho_{p z}(E)-$ $\frac{1}{2}\left[\rho_{p x}(E)+\rho_{p y}(E)\right]$ which determines the EFG. The individual contributions $\rho_{p z}$ (dashed line) and $\rho_{p x}=\rho_{p y}$ (dotted line) are also given.

by a simple hybridization model starting from the single defects. A single $\mathrm{Cd}^{2-}$ acceptor has an occupied $T_{2}$ level (in the notation of the $T_{d}$ symmetry group of the single defect) at the bottom of the energy gap, while the single $D$ donor atom has an $A_{1}$ level at the top of the band gap. Because of the $C_{3 v}$ symmetry of the pair the $T_{2}$ level splits up into a symmetric $A_{1}$ level, which can be occupied by two electrons, and into an $E$ level, which can take up four electrons. The $E$ state consists, locally at the $\mathrm{Cd}$ site, of the more or less unperturbed $p_{x}$ and $p_{y}$ states of the $\mathrm{Cd}^{2-}$ acceptor, which for symmetry reasons cannot hybridize with the $A_{1}$ state of the donor atom and form a deep level at $0.18 \mathrm{eV}$ above the valence bands (the calculated band gap of $\mathrm{Si}$ is $0.49 \mathrm{eV}$ ). In contrast to this, the new $A_{1}$ states of the $C_{3 v}$ symmetry group essentially consist of two hybrids of the $s$ and $p_{z}$ orbitals of the Cd atom and the $A_{1}$ orbital of the donor. However, only the bonding hybrid of these $A_{1}\left(C_{3 v}\right)$ states is occupied, which is, due to hybridization, shifted down into the valence band (see Fig. 2) while the corresponding antibonding intensity is shifted up into the conduction band. For the EFG it is most important that the unoccupied $A_{1}$ antibonding states in the conduction band contain, due to hybridization, also a $p_{z}$ contribution of the $\mathrm{Cd}$ atom, so that locally the $\mathrm{Cd} p_{z}$ charge is decreased and transferred into $A_{1}$ charge at the donor site, while the $p_{x}$ and $p_{y}$ charges are practically unaffected. Therefore the loss of the $p_{z}$ charge leads to an anisotropic $p$-charge distribution, giving rise to an EFG at the $\mathrm{Cd}$ site. Since the hybridization increases with increasing donor size, so does the EFG, reflecting the increased loss of the $p_{z}$ charge of the $\mathrm{Cd}$ atom.

$[C d D]^{0}$ complexes in Si. - In PAC experiments with different donor concentrations and very high annealing temperatures, Achtziger and Witthuhn [5] found strong evidence that the $\mathrm{Cd} D$ pairs in silicon form electrically deep centers with two different charge states, i.e., $[\mathrm{Cd} D]^{-}$ and $[\mathrm{Cd} D]^{0}$, leading to two different EFGs. Though the sign of the EFG cannot be determined by the experiments, by assuming the same sign for both charge states the resulting change in the EFG, $\Delta_{\mathrm{EFG}}$, turned to be more or less independent of the donor type (about $145 \mathrm{MHz}$ ).

The above-mentioned results are unequivocally confirmed by our calculations. For this we have performed spin polarized calculations, with the majority $E$ level filled by two electrons and the minority level by only one electron, without reducing the $C_{3 v}$ symmetry $(\eta=0)$. This leads for all three donor types to a small magnetic moment of $0.07 \mu_{B}$ and a small hyperfine field of $8 \mathrm{kG}$ at the $\mathrm{Cd}$ site. The resulting EFGs for both the ideal and the relaxed positions are shown in Table I.

In the relaxed geometry the sign of the EFGs $V_{z z}$ remains the same (compared to $[\mathrm{Cd} D]^{-}$) and the differences, $\Delta_{\mathrm{EFG}}$, are 135,160 , and $159 \mathrm{MHz}$ for $\mathrm{CdP}, \mathrm{CdAs}$, and $\mathrm{CdSb}$ pairs, respectively, which is in good agreement with the experimental data. On the one hand, the difference results from the different charge and on the other hand from the change in the forces on the atoms. The reduction of charge mainly affects the relaxations of the $\mathrm{Cd}$ atom, so that in the case of the $\mathrm{P}$ donor the $\mathrm{Cd}$ atom moves now slightly inward by $1.7 \%$ of the nn distance, while the $\mathrm{P}$ atom moves outward about $5.9 \%$ (instead of $4.5 \%$ ) of the nn distance. For the As donor the situation changes only a little, since the As donor moves outward by $6.3 \% \mathrm{nn}$ distance like the $\mathrm{P}$ donor, but the $\mathrm{Cd}$ acceptor shifts outward by only $0.9 \%$ (instead of $4.5 \%$ ) of nn distance. In the case of the $[\mathrm{CdSb}]^{0}$ pair the acceptor displacements are-compared to the $[\mathrm{CdAs}]^{0}$ pair-increased $(10.2 \%$ outward) and the donor relaxations decreased (2.3\% outward). The lowering of the EFG can be understood again in terms of the abovementioned hybridization model. Since one electron is taken out of the $E$ state, the overcompensation of $\rho_{p z}$ by $\frac{1}{2}\left[\rho_{p x}+\rho_{p y}\right]$, leading to the strong anisotropy of the Cd $p$ charge in $[\mathrm{Cd} D]^{-}$, is reduced. Since the $E$ level itself is not affected by the hybridization, the change of the charge state of the defect leads to a donor independent change of the EFG.

$\left[C d D_{2}\right]^{0}$ trimers in $\mathrm{Si}$. - In the case of stronger doping with $\mathrm{P}$ and As donor atoms the PAC spectra yield [13] two additional frequencies for the nuclear coupling constants, \pm 195 and $\pm 238 \mathrm{MHz}$ for $\mathrm{P}$ and As, respectively. In both measurements the asymmetry parameter is $\eta=0.65$. Because of symmetry considerations the additional EFGs were attributed to a substitutional trimer along the [110] zigzag line as shown in Fig. 3. The calculated relaxed positions are shown in Fig. 3. The top left-hand portion of the picture shows the three-dimensional arrangement of the impurity atoms and their nearest neighbors. The righthand side shows the zigzag line with two neighboring host atoms in the (110) plane and the bottom left-hand 


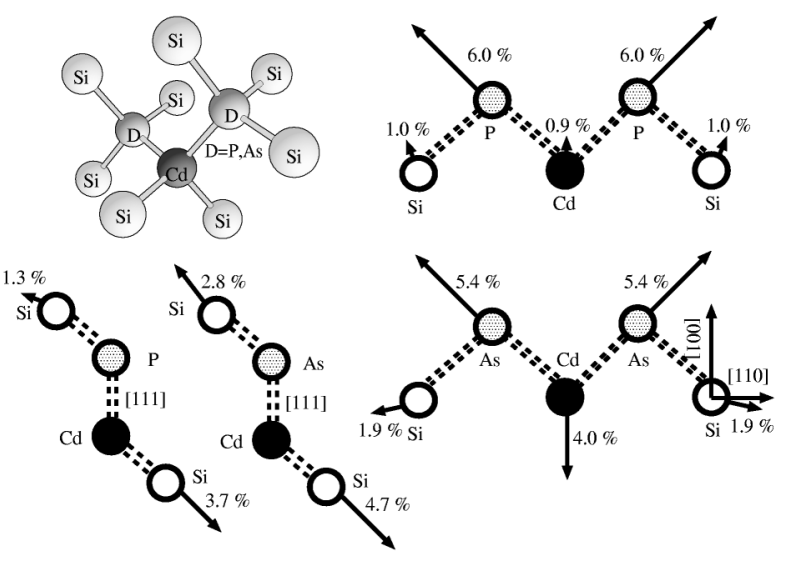

FIG. 3. Lattice relaxations for the defect atoms and for the nn atoms of $\operatorname{Cd} D_{2}(D=\mathrm{P}$, As $)$ in $\mathrm{Si}$. Shown are the three-dimensional arrangement of the $\operatorname{Cd} D$ complex (top, left hand), the (110) planes containing the $\operatorname{Cd} D$ pairs and the two representative Si neighbors (bottom left), and the (110) planes including the zigzag line (right hand), which contains the $\mathrm{Cd} D_{2}$ complexes and the two representative silicon neighbors. The relaxations are given in percentages of the nn distance.

portion the (110) plane to display the other neighboring host atoms. As in Fig. 1 the numbers given refer to the relative relaxations as a percentage of the nn distance. For the relaxed positions we calculate the nuclear quadrupole constants to be $\nu=+222 \mathrm{MHz}, \eta=0.68$ for $\left[\mathrm{CdP}_{2}\right]^{0}$ and $\nu=+246 \mathrm{MHz}, \eta=0.70$ for $\left[\mathrm{CdAs}_{2}\right]^{0}$. Both results are close to the experimental values. Especially the asymmetry parameter $\eta$ is well reproduced. The EFG lies in the [001] plane as expected from the symmetry. Both EFGs can be qualitatively understood as resulting from the superposition of the values for single $[\mathrm{Cd} D]^{-}$ pairs. For the single pairs the tensor of the EFG $\mathbf{Q}_{i j}$ can be written as $\mathbf{Q}_{i j}^{111}=-\left(V_{z z} / 2\right) \delta_{i j}+\frac{3}{2} V_{z z} e_{i}^{111} e_{j}^{111}$. The second pair is oriented along the direction [ $1 \overline{1} 11]$. The resulting tensor, $\mathbf{Q}_{i j}=\mathbf{Q}_{i j}^{111}+\mathbf{Q}_{i j}^{1 \overline{1} 1}$, consists of two equal eigenvalues $\left(V_{z z}\right.$ and $\left.-V_{z z}\right)$ and a vanishing one, so that the asymmetry parameter is $\eta=1$ and the absolute value of the two nonvanishing eigenvalues agrees with $V_{z z}$ of the single pair. Because of the electronic and geometric relaxations the superposition model is, of course, too simplified. There exists also a small component of the EFG in the [001] direction, which leads to the degeneracy breaking and thus to a lowering in $\eta$. This component also causes a small deviation of the absolute values of the EFG compared to the single pairs.

We performed self-consistent density-functional calculations of the electric field gradients of different acceptordonor complexes in silicon and germanium. Our results show that the EFG of the Cd probe atom is determined to a large extent by the lattice relaxations of the complex and the neighboring $\mathrm{Si}$ atoms. From this we conclude that the calculated lattice distortions give reliable information about the complicated geometrical structures of the defect complexes. The origin of the EFG can be qualitatively understood by a simple hybridization model and results from a loss of $p_{z}$ charge at the $\mathrm{Cd}$ site. The $[\mathrm{Cd} D]^{-/ 0}$ pairs in $\mathrm{Si}$ are proven to induce deep electrically active centers in the energy gap. The lowering of the EFG in the case of neutral pairs is shown to be a consequence of reloading the gap level and is nearly independent of the dopant due to the loss of the local $p_{x}$ and $p_{y}$ charges. The complexes $\left[\mathrm{CdP}_{2}\right]^{0}$ and $\left[\mathrm{CdAs}_{2}\right]^{0}$ produce almost the same EFGs as the single pairs, which can qualitatively be explained by a superposition of the single EFGs. Thus we obtain a consistent picture of the electronic and geometrical structure of acceptor-donor pairs in $\mathrm{Si}$ and $\mathrm{Ge}$ and, in particular, a simple understanding of the EFGs of the $\mathrm{Cd} D$ complexes measured by PAC experiments.

We thank H. R. Schober for providing his lattice statics code and for helpful discussions. This work was funded by the Federal Ministry for Science and Technology (BMBF, Contract No. 05606CJA3).

*Present address: Laboratory of Physics, Helsinki University of Technology, P.O. Box 1100, FIN-02015 HUT, Finland.

[1] Th. Wichert and M.L. Swanson, J. Appl. Phys. 66, 3026 (1989).

[2] Th. Wichert, M. Grübel, G. Keller, N. Schulz, and H. Skudlik, Appl. Phys. A 48, 59 (1989).

[3] Th. Wichert, in Semiconductors and Semimetals (Academic, New York, 1999), Vol. 51B, Chap. 6.

[4] D. Forkel, N. Achtziger, A. Baurichter, M. Deicher, S. Deubler, M. Puschmann, H. Wolf, and W. Witthuhn, Nucl. Instrum. Methods Phys. Res., Sect. B 63, 217 (1992).

[5] N. Achtziger and W. Witthuhn, Phys. Rev. B 47, 6990 (1993).

[6] P. Blaha, K. Schwarz, and P. H. Dederichs, Phys. Rev. B 37, 2792 (1988).

[7] K. Schwarz, C. Ambrosch-Draxl, and P. Blaha, Phys. Rev. B 42, 2051 (1990).

[8] S. H. Vosko, L. Wilk, and M. Nusair, J. Can. Phys. 58, 1200 (1980).

[9] J. Korringa, Physica (Utrecht) 13, 392 (1947).

[10] W. Kohn and N. Rostoker, Phys. Rev. 94, 111 (1954).

[11] P. J. Braspenning, R. Zeller, A. Lodder, and P.H. Dederichs, Phys. Rev. B 29, 703 (1984).

[12] M. Asato, A. Settels, T. Hoshino, T. Asada, S. Blügel, R. Zeller, and P.H. Dederichs, Phys. Rev. B 60, 5202 (1999).

[13] N. Papanikolaou, R. Zeller, P.H. Dederichs, and N. Stefanou, Phys. Rev. B 55, 4157 (1997).

[14] A. Settels, Ph.D. thesis, RWTH Aachen, 1999 (unpublished). 